\title{
Levsen, Sonja: Autorität und Demokratie. Eine Kulturgeschichte des Erziehungswandels in Westdeutschland und Frankreich, 1945-1975, 711 S., Wallstein, Göttingen 2019.
}

\section{Till Kössler}

Angenommen: 5. Januar 2021 / Online publiziert: 21. Januar 2021

(C) Der/die Autor(en) 2021

Erziehungsfragen werden von der Zeitgeschichte oft stiefmütterlich behandelt, erfuhren aber nach 1945 eine hohe Aufmerksamkeit und bildeten einen wichtigen Motor gesellschaftlichen und kulturellen Wandels. Diese Beobachtung steht am Ausgangspunkt der vorliegenden exzellenten Studie von Sonja Levsen, die dem Zusammenhang von Erziehung und Demokratie in der Bundesrepublik Deutschland und Frankreich zwischen Kriegsende und der Mitte der 1970er Jahre nachspürt. Die auf einer Freiburger Habilitationsschrift beruhende Arbeit unternimmt eine Neuinterpretation der Demokratieentwicklung in Westdeutschland, die gemeinhin als Überwindung autoritärer Strukturen und Mentalitäten sowie zugleich als Annäherung an ein westliches Modell von Demokratie beschrieben wird. Gegen diese verbreitete Deutung, die Demokratisierung als einen realhistorischen und messbaren Prozess versteht, richtet sich die Untersuchung mit einem Plädoyer für eine Historisierung der zentralen Begriffe Demokratie und Autorität.

In der Auswertung einer beeindruckenden Vielfalt zeitgenössischer Publikationen - pädagogische Fachjournale, Monografien sowie Jugend- und Elternzeitschriften - und Quellen der Bildungsadministrationen kann die Autorin deutliche Unterschiede in den Füllungen und Verwendungsweisen dieser Begriffe aufzeigen. Zunächst wurden Autorität und Demokratie nach 1945 keineswegs zwangsläufig als Gegensätze gefasst. Vielmehr stellte die Denkfigur einer ,autoritären“ Prägung der Gesellschaft, die eine Demokratisierung verhindere, eine bundesdeutsche Besonderheit dar, die ihren Ursprung in einer sozialpsychologischen Deutung des Nationalsozialismus durch amerikanische und exilierte deutsche Sozialwissenschaftler in den 1930er und 1940er Jahren hatte. Deren Thesen einer autoritären Formung der deutschen Gesellschaft als Grundbedingung nationalsozialistischer Herrschaft gewann nach Kriegsende lagerübergreifend an Einfluss. Demokratiefragen erschienen in dieser Perspektive immer auch als Erziehungsfragen und umgekehrt. Nach dieser Lesart konnten Frontalunterricht und Körperstrafen ebenso als Ausdruck eines Demokratiedefizits verstanden werden wie eine rigide ,repressive' Sexualerziehung.

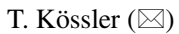

Martin-Luther-Universität Halle-Wittenberg, Halle, Deutschland

E-Mail: till.koessler@paedagogik.uni-halle.de 
Es ist eine große Stärke des Buches, diese Geschichte von Autorität, Erziehung und Demokratie nicht nur anhand der pädagogischen und bildungspolitischen Höhenkammliteratur zu verfolgen, sondern zudem wichtige Praxisfelder in den Blick zu nehmen und deren Wandel nachzuzeichnen. Im Mittelpunkt stehen das höhere Schulwesen, mit Seitenblicken auf Jugendverbände und Familie, sowie einige klug ausgewählte Themenfelder - neben Strafpraktiken und Sexualerziehung auch die Rolle von Politik an der Schule und die Schülerbewegung um und nach „1968“.

Der Vergleich mit Frankreich fördert einige frappierende Erkenntnisse zutage. In Frankreich nahmen die Erziehungsdebatten einen deutlich anderen Verlauf als in der Bundesrepublik. Hier bildete die Kriegsniederlage gegen Nazi-Deutschland im Jahr 1940 den Ausgangspunkt einer bildungspolitischen Reformströmung, die die Kapitulation auf das Versagen einer vermeintlich kastenartig abgeschotteten Elite zurückführte. Demgegenüber sollte nach Kriegsende über eine Ausweitung des Bildungszugangs eine Diversifizierung der nationalen Führungsschicht erreicht werden. Mit „Demokratisierung“ war diese Neuordnung von Bildungsaufstieg und Elitenbildung gemeint, kaum dagegen die Veränderung von Erziehungsformen und Lehrinhalten, die bis in die 1970er Jahre hinein weitgehend unproblematisch erschienen.

Angesichts dieser Befunde kommt die Autorin zu dem Schluss, dass es einen bundesdeutschen Sonderweg der Demokratieentwicklung gab. Auch im Verlauf der 1960er Jahre kam es nicht zu einer Annäherung im Zeichen einer Europäisierung. Im Gegenteil, die Schulkulturen beider Länder begannen sich immer deutlicher voneinander zu unterscheiden. Vor diesem Hintergrund formte sich auch „1968“ im Bildungsbereich und an den Schulen beider Länder unterschiedlich aus. Während in Frankreich unter dem starken Einfluss der kommunistischen Partei ökonomische, antikapitalistische Forderungen dominierten, gewannen in der Bundesrepublik Ideen einer ,antiautoritären“ Emanzipation als Veränderung individueller Lebenspraxis überragende Bedeutung.

Insgesamt demonstriert die präzise argumentierende und gut lesbare Studie eindrucksvoll die Erkenntnispotenziale erziehungshistorischer Zugänge. Sie lädt dabei sicherlich auch zu Nachfragen ein. So entfaltet die These eines bundesdeutschen pädagogischen Sonderwegs in Bezug auf die pädagogischen Reformmilieus eine hohe Plausibilität - auch weil sich die Zeitgenossen kaum für die Diskussionen im jeweiligen Nachbarland interessierten. Es wäre jedoch weiterer Untersuchung wert, wie die ,antiautoritären' Deutungs- und Handlungsmuster mit einer transnationalen Medienund Konsumkultur interagierten, die immer auch Bilder, guter' Erziehung über nationale Grenzen hinweg transportierte und zugleich Gesellschaftsschichten erreichte, die den akademischen Reformmilieus vermutlich eher distanziert gegenüberstanden. Solche weitergehenden Überlegungen unterstreichen den Wert der Studie, die gängige Narrative einer Demokratisierung der westdeutschen Gesellschaft produktiv auf den Prüfstand stellt und zu einer grundlegenden Reflexion über Entwicklungspfade der bundesdeutschen und europäischen Zeitgeschichte anregt. Es ließe sich gewiss diskutieren, inwiefern es für konkrete Reformdynamiken einen Unterschied machte, ob es sich bei den diagnostizierten ,,autoritären“ Traditionen um Tatsachen oder eine kulturelle Konstruktion handelte. Doch das erscheint müßig gegenüber der grundlegenden und auch aktuell höchst bedeutsamen Einsicht, dass Demokratieerziehung zu unterschiedlichen Zeiten höchst Unterschiedliches bedeuten konnte und kann. 
Funding Open Access funding enabled and organized by Projekt DEAL.

Open Access Dieser Artikel wird unter der Creative Commons Namensnennung 4.0 International Lizenz veröffentlicht, welche die Nutzung, Vervielfältigung, Bearbeitung, Verbreitung und Wiedergabe in jeglichem Medium und Format erlaubt, sofern Sie den/die ursprünglichen Autor(en) und die Quelle ordnungsgemäß nennen, einen Link zur Creative Commons Lizenz beifügen und angeben, ob Änderungen vorgenommen wurden.

Die in diesem Artikel enthaltenen Bilder und sonstiges Drittmaterial unterliegen ebenfalls der genannten Creative Commons Lizenz, sofern sich aus der Abbildungslegende nichts anderes ergibt. Sofern das betreffende Material nicht unter der genannten Creative Commons Lizenz steht und die betreffende Handlung nicht nach gesetzlichen Vorschriften erlaubt ist, ist für die oben aufgeführten Weiterverwendungen des Materials die Einwilligung des jeweiligen Rechteinhabers einzuholen.

Weitere Details zur Lizenz entnehmen Sie bitte der Lizenzinformation auf http://creativecommons.org/ licenses/by/4.0/deed.de.

\title{
Defrance, Corine/Herrmann, Tanja/Nordblom, Pia (Hrsg.): Städtepartnerschaften in Europa im 20. Jahrhundert, 359 S., Wallstein, Göttingen 2020.
}

\author{
Nina Szidat \\ Online publiziert: 21. Dezember 2020 \\ (C) Der/die Autor(en) 2020
}

Eine Europakarte mit den Namen verschiedener Groß- und Hauptstädte ziert den Umschlag des vorliegenden Sammelbandes. Dass Marseille beispielsweise in Schottland zu finden ist, stellt jedoch keinen Layoutfehler dar, sondern spielt auf die Lage der Partnerstadt Glasgow an. Somit verweist die Darstellung bereits auf den Gegenstand der Publikation und das Netz an transnationalen Bezügen, das über Städtepartnerschaften nach dem Zweiten Weltkrieg in ganz Europa entstand.

Der auf eine Tagung des Arbeitsbereichs Zeitgeschichte der Johannes GutenbergUniversität Mainz und des französischen LabEx EHNE (Écrire une Histoire Nouvelle de l'Europe) im Oktober 2015 zurückgehende Band verfolgt das Ziel, ,die Entstehung und die Geschichte der Städtepartnerschaften in Europa im 20. Jahrhundert

\footnotetext{
N. Szidat $(\bowtie)$

Universität Duisburg-Essen, Essen, Deutschland

E-Mail: nina.szidat@uni-due.de
} 